\title{
Aspects médico-légaux de la prolifération des médicaments destinés au traitement des troubles de l'érection
}

\section{Forensic aspects of therapies for erectile dysfunction}

Pascal KINTZ*, Marion VILLAIN, Julie SIMONIN, Vincent CIRIMELE

X'pertise Consulting, Laboratoire ChemTox, 3, rue Grünïnger - 67400 Illkirch - France

*Auteur à qui adresser la correspondance : Pascal KINTZ, X'pertise Consulting, Laboratoire ChemTox, 3, rue Grüninger - 67400 Illkirch - France - Tél : + 33390403332 - Fax : +33 390400541

E-mail : pascal.kintz@wanadoo.fr

(Reçu le 15 janvier 2006 ; accepté le $1^{\text {cr fémrier 2006) }}$

\section{RÉSUMÉ}

Homme de plus de 40 ans, ayant envie d'une vie sexuelle plus épanouie et nostalgique de ses performances érotiques d'antan. Voilà le cour de cible des laboratoires proposant un médicament destiné à traiter la dysfonction érectile. Selon. les services marketing, cet homme fait partie des « vital. sexuel ».

Les chiffres français, non vérifiés par les statistiques officielles, rapportent quelque trois millions d'hommes en panne plus ou moins régulière d'érection, d'origine organique ou psychologique.

A ce jour, trois médicaments sont inscrits à la Pharmacopée française, le sildénafil (Viagra ${ }^{\oplus}$, Pfizer), le tadalafil (Cialis ${ }^{\oplus}$, Lilly-Icos) et le plus récent, le vardenafl. (Levitra ${ }^{\circledR}$, Bayer). Sur le plan pharmacologique, ces molécules sont des inhibiteurs sélectifs de la phospho-diestérase 5 (PDE5), qui joue un rôle essentiel dans la relaxation des corps caverneux. L'efficacité du produit serait de l'ordre de $70 \%$, avec un indice de satisfaction des utilisateurs entre 48 et $78 \%$. Les effets secondaires de ces produits incluent des maux de tête, des rougeurs de la face et une perturbation des couleurs. La

\section{SUMMARY}

The drug sildenafil (Viagra ${ }^{\ominus}$, Pfizer) and more recently tadalafil (Cialis ${ }^{\oplus}$, Lilly-Icos) and vardenafil (Levitra ${ }^{\oplus}$, Bayer) have drown public attention to aphrodisiacs. The search for such sustances dates back millenia. It is admitted that in France, about 3 million men are suffering from erectile dysfunction.

The physiologic mechanism of erection of the penis involves release of nitric oxide (NO) in the corpus cavemosum during sexual stimulation. NO then activates the enzyme guanyle cyclase, which results in increased levels of cyclic guanosine monophosphate (cGMP), producing smooth muscle relaxation in the corpus cavernosum and allowing inflow of blood. The drugs have no direct relaxant effect, but enhance the effect of NO by inhibiting phosphodiesterase type 5 (PDE5), which is responsible for degradation of cGMP. Efficacity of the drugs is about $70 \%$, with about 48 to $78 \%$ of satisfaction.

Adverse effects associated with these drugs include hypotension, tachycardia, headhache, flushing, blurred vision, dyspepsia and musculoskeletal pain. They may potentiate the 
prise de médicament provoque un relâchement artériel et une baisse de la pression sanguine, ce qui engendre, sous peine de collapsus fatal, une contre-indication de l'absorption simultanée de produits abaissant la pression artérielle, conme les antihypertenseurs (surtout les dérivés nitrés) et les aphrodisiaques de type poppers.

Sur le plan analytique, le dosage par chromatographie liquide apparaît comme la méthode la plus satisfaisante.

Ce laboratoire analyse dans le sang, les urines et les cheveux les 3 substances actives par LC-MS/MS après extraction en. milieu alcalin $(\mathrm{pH} 9,5)$ par un mélange dichlorométhane/isopropanol/n-heptane (25/10/65). Les limites de quantification. sont largement suffisantes pour les besoins de la toxicologie, soit $5 \mathrm{ng} / \mathrm{ml}$ et $25 \mathrm{pg} / \mathrm{mg}$.

Sur le plan médico-légal et judiciaire, la littérature rapporte plusieurs décès innpliquant le sildénafil (en association) mais il ne semble pas que les deux autres produits aient été observés lors d'une issue fatale. L'absence de composante psychoactive ne conduit pas à un détoumement d'usage hors du contexte sexuel.

Enfin, il est désormais reconnu que le sildénafil et ses congénères sont les médicaments les plus contrefaits, en particulier sur Internet, où environ $50 \%$ des produits font partie des circuits illicites.

\section{MOTS-CLÉS}

Troubles de l'érection, sildénafil, tadalafil, vardénafil, contrefaçon.

\section{Introduction}

La recherche et l'emploi d'aphrodisiaques ne sont pas une attitude nouvelle. Cette pratique est vieille de plusieurs millénaires, puisque l'homme apparaît sur ce sujet sensible comme un éternel insatisfait. Les aphrodisiaques peuvent être répertoriés en 3 genres, en fonction de leur mode d'action (1) : ceux qui augmentent la libido, comme l'ambreine ou la bufoténine, ceux qui augmentent le plaisir sexuel, comme la cantharidine et enfin ceux qui augmentent l'intensité de ce plaisir, comme le ginseng.

Parmi ces produits de la puissance sexuelle, le sildenafil et ses dérivés (figure 1) ont une place à part. Produits miracles destinés à traiter près de 3 millions de Français souffrants de troubles de l'érection d'origine organique ou psychologique, ces substances font désormais l'objet d'une extraordinaire banalisation.

A ce jour, trois médicaments sont inscrits à la Pharmacopée française, le sildénafil (Viagraß, Pfizer), le tadalafil (Cialis@, Lilly-Icos) et le plus récent, le vardenafil (Levitraß, Bayer).

L'objet de cet article est de faire le point sur les aspects médico-légaux liés à l'usage détourné de ces principes actifs. hypotensive effects of co-administered organic nitrates. Best analytical results are obtained when liquid chromatography is performed. This laboratory is using LC-MS/MS after alkaline ( $p H$ 9.5) extraction using dichloromethane/isopropanol/n-heptane (25/10/65). Limits of quantitation are in the range $5 \mathrm{ng} / \mathrm{ml}$ or $25 \mathrm{pg} / \mathrm{mg}$ for blood/urine and hair; respectively.

At this time, several sildenafil-related deaths have been published in the scientific literature but no case involving the two other drugs seems available.

About $50 \%$ of the drugs proposed on the black market or via Internet are counterfeit.

\section{KEY-WORDS}

Erectile dysfunction, sildenafil, tadalafil, vardenafil, counterfeit drug.<smiles>CCCc1nn(C)c2c(=O)[nH]c(-c3cc(S(=O)(=O)N4CCN(C)CC4)ccc3OCC)nc12</smiles><smiles>CCCc1nc(C)c2c(=O)[nH]c(-c3cc(S(=O)(=O)N4CCN(CC)CC4)ccc3OCC)nn12</smiles><smiles>CN1CC(=O)N2NC(c3ccc4c(c3)OCO4)c3[nH]c4ccccc4c3CC2C1</smiles>

Tadalafi1, CIALIS*

Figure I : Structures chimiques des 3 médicaments. 


\section{Pharmacologie}

Le mécanisme physiologique de l'érection de pénis est lié à la libération d'oxyde nitrique (NO) dans le corpus cavernosum lors de la stimulation sexuelle. Le NO va alors activer une enzyme, la guanylate cyclase dont le résultat va conduire à une augmentation des concentrations de guanosine monophosphate cyclique (cGMP), ce qui va permettre une relaxation des muscles lisses du pénis et donc un afflux de sang. Le sildénafil et ses dérivés n'ont pas d'effet direct sur la relaxation musculaire, mais ils augmentent les effets du NO en inhibant la phosphodiestérase de type 5 (PDE5), qui est responsable de la dégradation de la cGMP dans le corpus cavernosum.

Lorsque la stimulation sexuelle produit une libération de NO, l'inhibition de la PDE5 par le sildénafil ou ses dérivés induit une augmentation de cGMP, responsable de la relaxation des muscles lisses et donc d'une arrivée de sang dans le corpus cavernosum (figure 2).

Ces médicaments, aux doses recommandées, n'ont donc pas d'effet en absence de stimulation sexuelle (2). Ils n'augmentent pas la qualité des érections chez des sujets ne souffrant pas de trouble érectile et leur intérêt est donc nul chez le sujet jeune, sauf à raccourcir le délai réfractaire entre 2 érections.

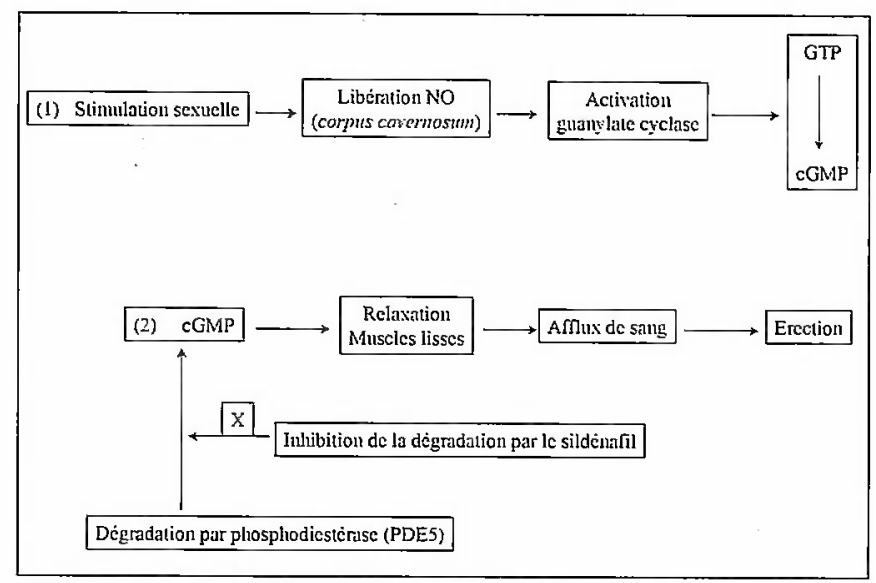

Figure 2 : Mécanisne d'action du sildénafil et de ses dériyés.

Le sildénafil est rapidement absorbé par voie orale, avec une biodisponibilité absolue de l'ordre de $40 \%$. Les concentrations plasmatiques maximales, de l'ordre de $450 \mathrm{ng} / \mathrm{ml}$ pour une dose de $100 \mathrm{mg}$ sont atteintes en 30 à $120 \mathrm{~min}$. Le volume de distribution est de l'ordre de 1 à 2 l/kg. Environ $90 \mathrm{~min}$ après l'administration orale, moins de $0,001 \%$ de la dose est retrouvée dans le sperme.

Le principe actif est principalement métabolisé au niveau hépatique par les cytochromes P450 3A4 (voie majoritaire) et P450 2C9 (voie mineure) et est transformé en métabolite actif, le $\mathrm{N}$-desméthyl sildénafil. Il existe donc des interactions avec les inhibiteurs classiques du CYP450 3A4 (érythromycine, kétocanazole, itraconazole ...). La demi-vie terminale du sildénafil et de son métabolite est de l'ordre de 4 heures. Environ $80 \%$ de la dose sont excrétées dans les feces et $13 \%$ dans les urines. Il existe des métabolites mineurs obtenus après pyrazole $\mathrm{N}$-déméthylation, $\mathrm{N}, \mathrm{N}$ '-deéthylation, oxydation du noyau pipérazine et hydroxylation aliphatique (3).

Des molécules plus actives sur la PDE5, et donc dosées plus faiblement ont été mises sur le marché plus récemment. Il s'agit du vardénafil (4) et du tadalafil (5), dont l'usage semble moins fréquent par rapport au sildénafil.

\section{Effets secondaires - contre indications}

Les inhibiteurs de la PDE5 et les nitrates augmentent les concentrations de cGMP dans la circulation centrale. Cette combinaison est contre-indiquée puisqu'elle conduit à majorer la vasodilatation et peut induire une hypotension aux conséquences désastreuses.

Néanmoins, la plupart des études cliniques rapportent que le sildénafil est bien toléré à concentration thérapeutique et que les effets secondaires sont essentiellement de type mal de tête (16\%), face rouge et chaude $(10 \%)$ et nausées $(2 \%)$. L'incidence des malaises liés à une hypotension est inférieure à $2 \%$. L'emploi concomitant de molécules adrenergiques, de diurétiques, d'inhibiteurs de l'enzyme de conversion ou d'antagonistes calciques ne semble pas conduire à des complications (6).

Du fait d'une faible activité sur la PDE6, localisée dans le cône des photo-récepteurs oculaires, des troubles de la vision (couleur bleutée, manque de contours nets ...) ont été rapportés (7).

Une interaction particulière a fait l'objet de plusieurs publications, celle entre le sildenafil et le jus de pamplemousse (8). En effet, 200 à $300 \mathrm{ml}$ de jus sont susceptible d'inactiver de façon irréversible les cytochromes intestinaux CYP450 3A4, ce qui va augmenter la Cmax (jusqu' à $42 \%$ ) mais pas l'aire sous la courbe $(8,9)$.

\section{Aspects analytiques}

Depuis la mise sur le marché du sildénafil, l'essentiel des méthodes de dosage publiées dans la littérature fait 
appel à la chromatographie liquide.

La différence entre les travaux est liée à l'emploi de détecteurs plus ou moins spécifiques, comme l'UV (8), la spectrométrie de masse (10) ou la spectrométrie de masse en tandem $(11,12)$. S'il existe une méthode de LC-MS/MS applicable aux fluides biologiques pour le tadalafil (13), il ne semble pas que quelque chose ait été décrit pour le vardénafil.

La méthode ci-dessous est utilisée au laboratoire.

\section{Extraction du sang et des urines}

A $0,5 \mathrm{ml}$ de sang ou d'urine, sont ajoutés $5 \mathrm{ng}$ de diazépam- $\mathrm{d}_{5}$, utilisé comme standard interne et $1 \mathrm{ml}$ de tampon chlorure d'ammonium ajusté à $\mathrm{pH}$ 9,5. Le mélange est alors extrait par $5 \mathrm{ml}$ de dichlorométhane/isopropanol/n-heptane $(25 / 10 / 65, \mathrm{v} / \mathrm{v} / \mathrm{v})$. Après agitation et centrifugation, la phase organique est évaporée à sec et l'extrait est repris par $50 \mu \mathrm{l}$ d'acétonitrile/tampon formiate $2 \mathrm{mM}(20 / 80, \mathrm{v} / \mathrm{v})$.

\section{Extraction des cheveux}

Après décontamination de la mèche de cheveux par du dichlorométhane ( $2 \times 5 \mathrm{ml}$ pendant $2 \mathrm{~min}$ ), les cheveux sont segmentés (en fonction des données du dossier, de la date des faits, de la date du prélèvement et sur la base d'une vitesse de pousse de $1 \mathrm{~cm} / \mathrm{mois}$ ) puis chaque segment est coupé en petits fragments de 1 à $2 \mathrm{~mm}$. Environ $50 \mathrm{mg}$ sont incubés toute la nuit à $45^{\circ} \mathrm{C}$ dans $1 \mathrm{ml}$ de tampon chlorure d'ammonium $\mathrm{pH} 9,5$, en présence de $5 \mathrm{ng}$ de diazépam- $\mathrm{d}_{5}$ utilisé comme étalon interne (EI) et extraits par $5 \mathrm{ml}$ de dichlorométhane/isopropanol/n-heptane $(25 / 10 / 65, \mathrm{v} / \mathrm{v} / \mathrm{v})$. Après agitation (15 min) et centrifugation ( $10000 \mathrm{~g}$ pendant $15 \mathrm{~min}$ ), la phase organique est recueillie et évaporée dans un SpeedVac®. Le résidu est reconstitué dans $50 \mu \mathrm{l}$ d'acétonitrile/tampon formiate $2 \mathrm{mM}(20 / 80, \mathrm{v} / \mathrm{v})$.

\section{Analyse par LC-MS/MS}

Une fraction de $10 \mu \mathrm{l}$ de l'extrait est injectée dans la colonne (XTerra MS C18 3,5 $\mu \mathrm{m}, 100$ x 2,1 mm i.d.), protégée par un fritté de $1 \mathrm{~mm} \mathrm{C} 18$. Le cycle d'analyse de 20 min fait appel à un gradient $(20 \%$ acétonitrile / $80 \%$ tampon formiate $2 \mathrm{mM}+$ acide formique $0,1 \%$ jusqu'à un rapport $80 / 20 \%$ à $9 \mathrm{~min}$ ), avec un débit de $200 \mu \mathrm{l} / \mathrm{min}$. Le système HPLC est un Waters Alliance 2695.

La détection est réalisée par un Micromass Quattro Micro, spectromètre de masse en tandem équipé d'une interface de type ionspray à pression atmosphérique, l'instrument étant en mode ionisation positive. Les meilleurs résultats ont été obtenus avec une tension de capillaire de $3 \mathrm{kV}$, une température de source à $120^{\circ} \mathrm{C}$ et de désolvatation de l'azote à $350^{\circ} \mathrm{C}$ à un débit de $550 \mathrm{~L} / \mathrm{h}$. La pression en argon dans la cellule de collision était de 4 mbar.

Les données ont été enregistrées selon le mode multiple reaction monitoring (MRM). Les ions parents, ions fils, temps de rétention, tensions de cône et énergies de collision, optimisés pour l'ensemble des composés, sont présentés dans le Tableau I.

Les limites de quantification sont largement suffisantes pour les besoins de la toxicologie, soit $5 \mathrm{ng} / \mathrm{ml}$ et $25 \mathrm{pg} / \mathrm{mg}$.

Les figures 3 et 4 représentent respectivement un calibrateur urinaire surchargé par les 3 molécules à $50 \mathrm{ng} / \mathrm{ml}$ et un échantillon urinaire recueilli 8 heures après administration d'un comprimé de Cialis (tadalafil à $20 \mathrm{mg}$ ).

Tableau I : Paramètres de chromatographie et de spectronétrie de masse.

\begin{tabular}{|c|c|c|c|c|c|}
\hline Substance & $\begin{array}{c}\text { Temps de rétention } \\
\text { (min) }\end{array}$ & $\begin{array}{c}\text { Ion parent } \\
(\mathbf{m} / \mathbf{z})\end{array}$ & $\begin{array}{c}\text { Ions fils } \\
(\mathrm{m} / \mathrm{z})\end{array}$ & $\begin{array}{c}\text { Tension de cône } \\
\text { (V) }\end{array}$ & $\begin{array}{c}\text { Énergie de collision } \\
\text { (eV) }\end{array}$ \\
\hline \multirow{2}{*}{ Vardénafil } & \multirow{2}{*}{7,26} & \multirow{2}{*}{489,1} & 151,1 & 50 & 40 \\
\hline & & & 312,0 & 50 & 40 \\
\hline \multirow{2}{*}{ Sildénafil } & \multirow{2}{*}{8,02} & \multirow{2}{*}{475,0} & 57,8 & 40 & 45 \\
\hline & & & 99,6 & 40 & 30 \\
\hline \multirow{2}{*}{ Tadalafil } & \multirow{2}{*}{9,59} & \multirow{2}{*}{390,0} & 135,0 & 23 & 21 \\
\hline & & & 268,1 & 23 & 15 \\
\hline \multirow{2}{*}{ Étalon interne } & \multirow{2}{*}{10,69} & \multirow{2}{*}{289,9} & 154,0 & 40 & 32 \\
\hline & & & 198,1 & 40 & 32 \\
\hline
\end{tabular}




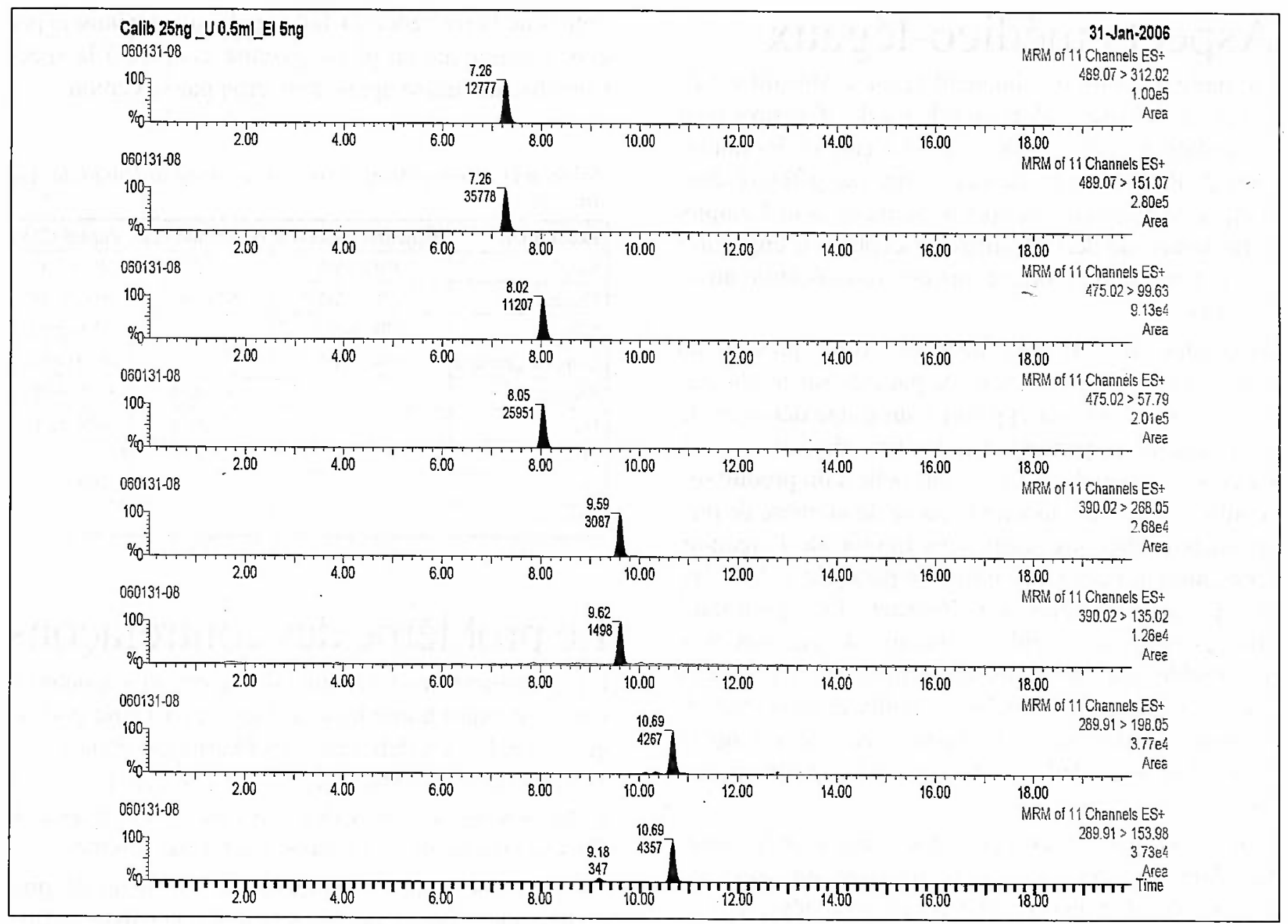

Figure 3 : Calibrateur urinaire dosé à $50 \mathrm{ng} / \mathrm{ml}$ de 3 médicaments. De haut en bas, successivement 2 ions fils du vardénafl, sildénafil, tadalafil et de l'étalon interne.

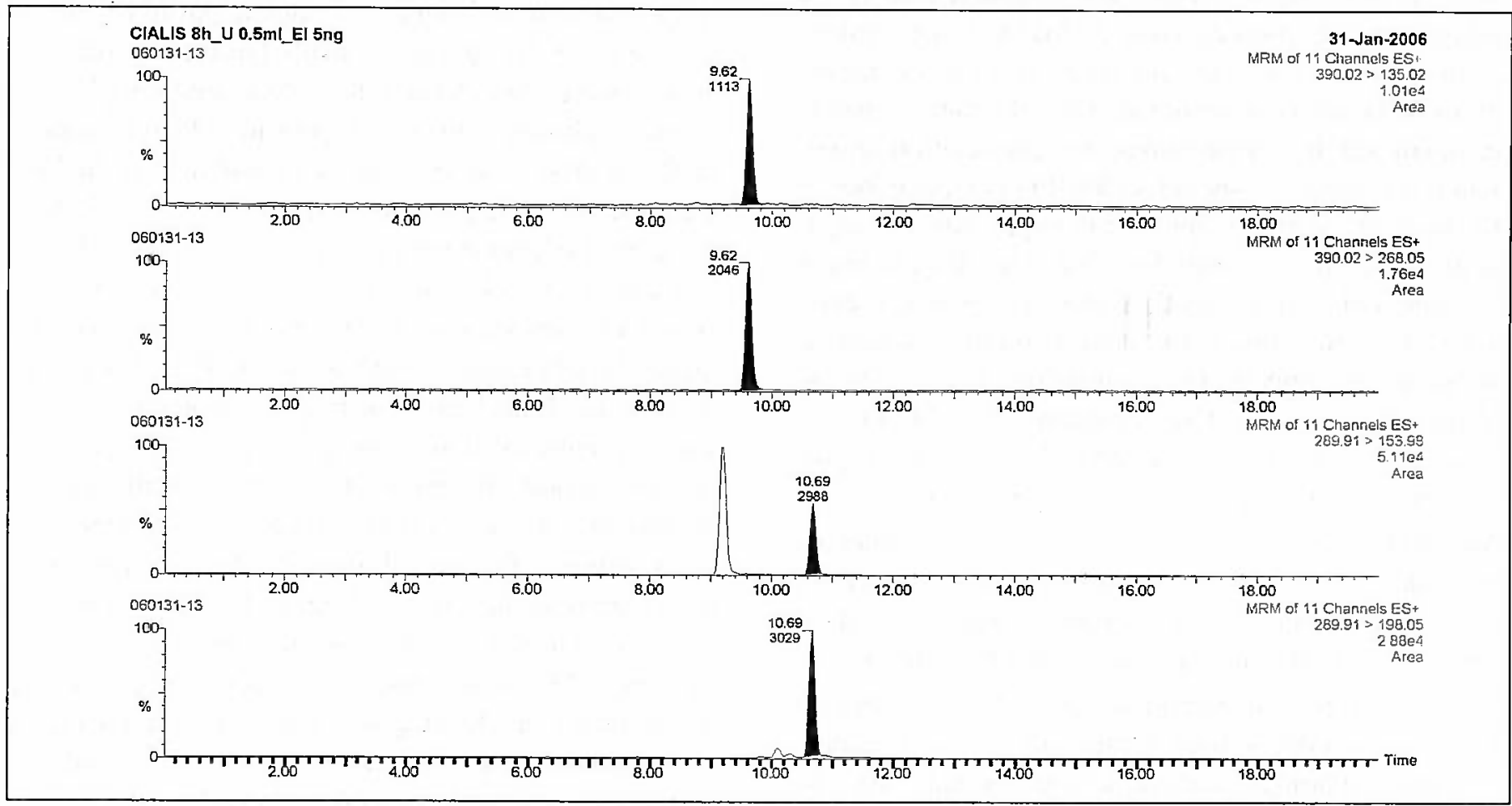

Figure 4 : Échantillon urinaire recueilli 8 heures après administration de 20 mg de Cialis. La concentration de tadalafil était de $46 \mathrm{ng} / \mathrm{ml}$. De haut en bas, successivement 2 ions fils du tadalafil et de l'étalon interne. 


\section{Aspects médico-légaux}

La durée d'action du sildénafil et du vardénafil est de l'ordre de 4 heures, alors qu'elle est de 36 heures pour le tadalafil. Les indices de sécurité d'emploi des inhibiteurs de PDE5 sont bons, même chez des patients souffrant de pathologie cardio-vasculaire et seul l'emploi concomitant de dérivés nitrés, susceptible d'engendrer une hypotension fatale est une contre-indication absolue (14).

Avec plus de 600000 médecins ayant prescrit du Viagra à plus de 16 millions de patients sur la planète, il était normal de voir apparaître un usage détourné du médicament, en particulier à des fins récréatives (15). La croyance populaire fait la part belle à un produit qui serait capable d'augmenter la durée de la phase de prééjaculation chez les sujets sans trouble de l'érection. L'essentiel de cette consommation parallèle se fait avec des produits achetés sur Internet. Les premières craintes liées aux accidents initiaux de patients avec des troubles cardiaques majeurs ont rapidement cédées sous la publication d'articles de synthèse présentant de manière satisfaisante une bonne sécurité d'emploi. C'est cette levée de l'anxiété qui a été la porte ouverte aux abus.

Dans une revue de 2005 (16), Swearingen et Klausner ont démontré que les sujets utilisant du sildénafil avaient plus de rapports sexuels non protégés que ceux qui n'en n'utilisent pas et que le pourcentage de maladies sexuellement transmissibles était le double dans la première population. Par ailleurs, un article alarmant a indiqué une mutation du virus du SIDA et une virulence plus importante chez un sujet utilisant en même temps de la méthamphétamine et du sildénafil. L'article terminait sur les implications de l'association stupéfiant (comme la cocaïne) et sildénafil (17). A ce jour, il n'existe pas d'étude épidémiologique sur ce sujet, même si certains rapports font état d'un détournement du sildénafil et l'association avec les produits classiques de la nuit comme l'ecstasy, le GHB, la kétamine ou encore les poppers (18), pour induire hallucinations, desinhibition sociale et augmentation de la libido. Les troubles de l'érection, liés à l'abus de stupéfiants (opiacés, amphétamines) pourraient être ainsi aussi corrigés. Plus anecdotique, une publication rapporte l'internement de 2 hommes pour comportement dangereux (envie d'homicide sur leur épouse) après consommation de sildénafil et manque de résultat probant (19).

Comme écrit précédemment, le sildénafil a été identifié dans plusieurs décès, tous impliquant des sujets males, avec une pathologie cardiaque sous-jacente $(10,20$, 21). Les concentrations post mortem sont très variables. Elles sont données dans le Tableau II. Il est à noter que la référence 21 fait appel à une technique par chromatographie en phase gazeuse couplée à la spectrométrie de masse après dérivation par silylation.

Tableau II : Concentrations post mortem en sildénafil dans la littérature.

\begin{tabular}{|l|c|c|c|}
\hline Échantillon & Dumestre-Toulet (10) & Weinmann (20) & Pagani (21) \\
\hline Sang & $105 \mathrm{ng} / \mathrm{ml}$ & & $40 \mathrm{ng} / \mathrm{ml}$ \\
\hline Urines & $246 \mathrm{ng} / \mathrm{ml}$ & $63 \mathrm{ng} / \mathrm{ml}$ & $9600 \mathrm{ng} / \mathrm{ml}$ \\
\hline Bile & $1206 \mathrm{ng} / \mathrm{ml}$ & & $999 \mathrm{ng} / \mathrm{ml}$ \\
\hline Contenu gastrique & $754 \mathrm{ng} / \mathrm{ml}$ & & $6840 \mathrm{ng} / \mathrm{ml}$ \\
\hline Encéphale & & $1,2 \mathrm{ng} / \mathrm{g}$ & $6430 \mathrm{ng} / \mathrm{g}$ \\
\hline Foie & & $74 \mathrm{ng} / \mathrm{g}$ & $5460 \mathrm{ng} / \mathrm{g}$ \\
\hline Muscle & & $0,5 \mathrm{ng} / \mathrm{g}$ & \\
\hline Coeur & & & $6100 \mathrm{ng} / \mathrm{g}$ \\
\hline Cheveux & $177 \mathrm{pg} / \mathrm{mg}$ & & \\
\hline
\end{tabular}

\section{Le problème des contrefaçons}

L'engouement des « produits de l'érection » a naturellement conduit à une mise à disposition facile pour le grand public. La délivrance en Pharmacie étant liée à une ordonnance médicale du fait de l'inscription de ces composés sur la liste I des substances vénéneuses, il s'avère plus facile de les commander par Internet.

Tout comme pour les stupéfiants, l'offre étant plus grande que la demande, les prix sont à la baisse. A titre d'exemple, le comprimé de sildénafil s'achetait 5,49 dollars en 1999 (22), alors qu'il est aujourd'hui proposé autour de 2 dollars. Comptant parmi les hits du vaste monde des spams, les mails faisant la promotion et la vente de la célèbre pilule bleue sont envoyés par millions chaque jour. C'est pourquoi Pfizer, dont le chiffre d'affaire sur le Viagraß a chuté de $7 \%$ en 2004 a décidé de s'attaquer aux vendeurs en ligne de faux Viagra®. Le laboratoire pense qu'environ 350000 sites vendent des versions illégales du médicament ou renvoient vers des sites qui le font. Pour enrayer la contrefaçon, le laboratoire a décidé d'employer les grands moyens aux États Unis. Une puce électronique associée à une antenne est désormais apposée sur l'étiquette de chaque conditionnement. Cette puce renferme un numéro spécifique et unique qui peut, via l'antenne être lue à distance. En effet, d'après Pfizer, près 'd'un comprimé sur deux achetés en ligne au Royaume Uni était faux. Ces données ont été confirmés par une large étude en Corée (23), où seulement $75 \%$ des comprimés vendus comme étant du Viagra ${ }^{\circledR}$ contenaient effectivement du sildénafil. Pour le Cialis® (tadalafil) illégal, les comprimés renfermaient à $66 \%$ du sildénafil. 


\section{Conclusion}

Produit miracle, ou médicament actif sur une pathologie difficile à avouer ?

La puissance de l'homme face aux difficultés anatomiques!

Le sildénafil, chef de file des inhibiteurs de la phosphodiestérase 5 est devenu une molécule banale. Les premiers rapports alarmants, liés à des décès brutaux, sont aujourd'hui oubliés et ces produits, tellement facile à se procurer sur Internet font l'objet d'un abus récréatif, en particulier en association avec les stupéfiants de type MDMA, GHB ou cocaïne. Et en plus, personne ne dit rien ...

\section{Références}

1. Sandroni P. Aphrodisiacs past and present : a historical review. Clin. Auton. Res. $2001 ; 11: 303-307$.

2. Mondaini N., Ponchietti R., Muir G.H., Montorsi F., Di Loro F., Lombardi G., Rizzo M. Sildenafil does not improve sexual function in men without erectile dysfunction but does reduce the postorgasmic refractory time. Int. J. Impot. Res. $2003 ; 15: 225-228$.

3. Walker D.K., Ackland M.J., James G.C., Muirhead G.J., Rance D.J., Wastall P., Wright P.A. Pharmacokinetics and metabolism of sildenafil in mouse, rat, rabbit, dog and man. Xenobiotica $1999 ; 29: 297-310$.

4. Bella A.J., Brock G.B. Tadalafil in the treatment of erectile dysfunction. Curr. Urol. Rep. $2003 ; 4$ : 472-478.

5. Jin J., Guo Y. Pharmacodynamics and pharmacokinetics of vardenafil in patients with erectile dysfunction. Zhonghua Nan Ke Xue $2004 ; 10$ : 711-716.

6. Zusman R.M., Morales A., Glasser D.B., Osterloh I.H. Overall cardiovascular profile of sildenafil citrate. Am. J. Cardiol. $1999 ; 83$ : 35C-44C.

7. Laties A., Sharlip I. Ocular safety in patients using sildenafil citrate therapy for erectile dysfunction. J. Sex Med. $2006 ; 3: 12-27$.

8. Lee M., Min D.I. Determination of sildenafil citrate in plasma by high performance liquid chromatography and a case for the potential interaction of grapefruit juice with sildenafil citrate. Ther. Drug Monit. 2001 ; 23 : 21-26.

9. Bailey D.G., Dresser G.K. Interactions between grapefruit juice and cardiovascular drugs. Am. J. Cardiovasc. Drugs $2004 ; 4$ : 281-297.

10. Dumestre-Toulet V., Cirimele V., Gromb S., Beloussoff T., Lavault D., Ludes B., Kintz P. Last performance with VIAGRA : post-mortem identification of sildenafil an dits metabolites in biological specimens including hair samples. Forensic Sci. Int. 2002 ; 126 : 71-76

11. Kim J., Ji H., Kim S.J., Lee H.W., Lee S.S., Kim D.S., Yoo M, Kim W.B., Lee H.S. Simultaneous determination of sildenafil an dits active metabolite UK-103,320 in human plasma using liquid chromatography-tandem mass spectrometry. J. Pharm. Biomed. Anal. 2003 ; $32:$ 317-322.
12. Eerkes A., Addison T., Naidong W. Simultaneous assay of sildenafil and desmethylsildenafil in human plasma using liquid chromatography-tandem mass spectrometry on silica column with aqueous-organic mobile phase. J. Chromatogr. B $2002 ; 768$ : 277-284.

13. Ramakrishna N.V., Vishwottam K.N., Puran S., Koteshwara M., Manoj S., Santosh M., Chidambara J., Wishu S., Sumatha B. Quantitation of taladafil in human plasma by liquid chromatography-tandem mass spectrometry with electrospray ionization. J. Chromatogr. B 2004 ; 809 : 243-249.

14. Rashid A. The efficacy and safety of PDE5 inhibitors. Clin. Cornerstone 2005; $7: 47-56$.

15. Alpert J.S. Viagra : the risk of recreational use. Am. J. Med. $2005 ; 118: 569-570$.

16. Swearingen S.G., Klausner J.D. Sildenafil use, sexual risk behavior, and risk for sexually transmitted diseases, including HIV infection. Am. J. Med. 2005 ; 118 : 571-574.

17. Markowitz M., Mohri H., Mehandru S. Infection with multidrug resistant, dual-tropic HIV-1 and rapid progression to AIDS : a case report. Lancet 2005 ; 365 : 1031 1038.

18. Smith K.M., Romanelli F. Recreational use and misuse of phosphodiesterase 5 inhibitors. J. Am. Pharm. Assoc. $2005 ; 45: 63-75$.

19. Mintz D. Unusual case report : nonpharmacologic effects of sildenafil. Psychiatr, Serv. $2000 ; 51: 674-675$.

20. Weinmann W., Bohnert M., Wiedemann A., Renz M., Lehmann N., Pollack S. Post-mortem detection and identifiçation of sildenafil (Viagra) an dits metabolites by LC/MS and LC/MS/MS. Int. J. Leg. Med. 2001 ; 114 : 252-258.

21. Pagani S., Mirtella D., Mencarelli R., Rodriguez D., Cingolani M. Postmortem distribution of sildenafil in histological material. J. Anal. Toxicol. $2005 ; 29$ : 254-258.

22. Bloom B.S., Iannacone R.C. Internet availability of prescription pharmaceuticals to the public. Ann. Intern. Med. $1999 ; 131: 830-833$.

23. Choi H., Lee J., Choi H., Woo S., Park Y, Chung H. The characteristics of illegal oral therapies for erectile dysfunction in Korea. Résumé 1-8 du 43ème congrès international du TIAFT, 29 août au 2 septembre 2005, Séoul, Corée. 